\title{
On Topological Derivatives for Contact Problems in Elasticity
}

\author{
S.M. Giusti · J. Sokołowski · J. Stebel
}

Received: 24 September 2012 / Accepted: 2 June 2014 / Published online: 2 July 2014 (C) The Author(s) 2014. This article is published with open access at Springerlink.com

\begin{abstract}
In this article, a general method for shape-topology sensitivity analysis of contact problems is proposed. The method uses domain decomposition combined with specific properties of minimizers for the energy functional. The method is applied to the static problem of an elastic body in frictionless contact with a rigid foundation. The contact model allows a small interpenetration of the bodies in the contact region. This interpenetration is modeled by means of a scalar function that depends on the normal component of the displacement field on the potential contact zone. We present the asymptotic behavior of the energy shape functional when a spheroidal void is introduced at an arbitrary point of the elastic body. For the asymptotic analysis, we use a nonoverlapping domain decomposition technique and the associated SteklovPoincaré pseudodifferential operator. The differentiability of the energy with respect to
\end{abstract}

Communicated by Günter Leugering.

\section{S. M. Giusti}

Departamento de Ingeniería Civil, Facultad Regional Córdoba, Universidad Tecnológica Nacional (UTN/FRC - CONICET), Maestro M. López esq. Cruz Roja Argentina, X5016ZAA Córdoba, Argentina e-mail: sgiusti@civil.frc.utn.edu.ar

\section{J. Sokołowski (凶)}

Laboratoire de Mathématiques, Institut Élie Cartan, UMR7502 (Université Lorraine, CNRS, INRIA), Université de Lorraine, B.P. 239, 54506 Vandoeuvre-lès-Nancy Cedex, France

e-mail: Jan.Sokolowski@univ-lorraine.fr

\section{J. Sokołowski}

Systems Research Institute of the Polish Academy of Sciences, Warsaw, Poland

\section{J. Stebel}

Institute of Mathematics of the Academy of Sciences of the Czech Republic, Žitná 25, Praha 1, 11567 Prague, Czech Republic

e-mail: stebel@math.cas.cz 
the nonsmooth perturbation is established, and the topological derivative is presented in the closed form.

Keywords Topological derivative $\cdot$ Static frictionless contact problem - Asymptotic analysis · Domain decomposition · Steklov-Poincaré operator

Mathematics Subject Classification (2000) $\quad$ 41A60 - 49J52 - 49Q10 - 35J50 . 35 Q93

\section{Introduction}

Topological asymptotic analysis [1-3] allows us to obtain an asymptotic expansion of a given shape functional of linear elasticity when a geometrical domain is singularly perturbed by the insertion of holes, inclusions, source-terms, or even cracks. The main concept arising from this analysis is the topological derivative. This derivative can be seen as a first-order correction of the unperturbed shape functional to approximate the perturbed shape functional. The topological derivative was rigorously introduced in [4]. Since then, this concept has proved extremely useful in the treatment of a wide range of problems; see, for instance, [5-11]. Concerning the theoretical development of the topological asymptotic analysis, besides the monograph [1], the reader is referred to [12-15].

Classically, contact problems are modeled by means of a nonpenetration condition between an elastic body and a rigid obstacle or foundation. This is known as a unilateral contact condition and is modeled using variational inequalities originated by the socalled Signorini Contact Problem. A less restrictive boundary condition on the contact region is obtained by considering the normal compliance model. In this kind of models, based on the assumption of small displacement, some interpenetration between the contacting bodies is allowed, and the boundary forces are given as a function of the interpenetration. However, such models allow an arbitrarily large interpenetration of the bodies in contact, which is physically rather unrealistic. Recently, a new class of models has been presented in [16], using less restrictive boundary conditions that allow small interpenetrations of the bodies. In such a model, the small interpenetration is governed by a function that depends on the normal component of the displacement field on the boundary of the potential contact region. Clearly, this is a nonlinear boundary condition for the contact problem, leading to a new class of variational inequalities.

The shape and topological asymptotic analysis for contact problems have been studied in [17-20]. In these works, the differentiability of the energy functional with respect to a singular perturbation has been developed for the usual boundary conditions in contact problems. Due to the nonlinear condition over the contact zone, the boundary value problem becomes nonsmooth. Therefore, nonsmooth analysis is necessary since the shape differentiability of solutions to contact problems is obtained only in the framework of Hadamard differentiability of metric projections onto polyhedric sets in appropriate Sobolev spaces.

In this work, we consider the asymptotic behavior of the energy shape functional when a spheroidal void is introduced at an arbitrary point of the elastic body. We 


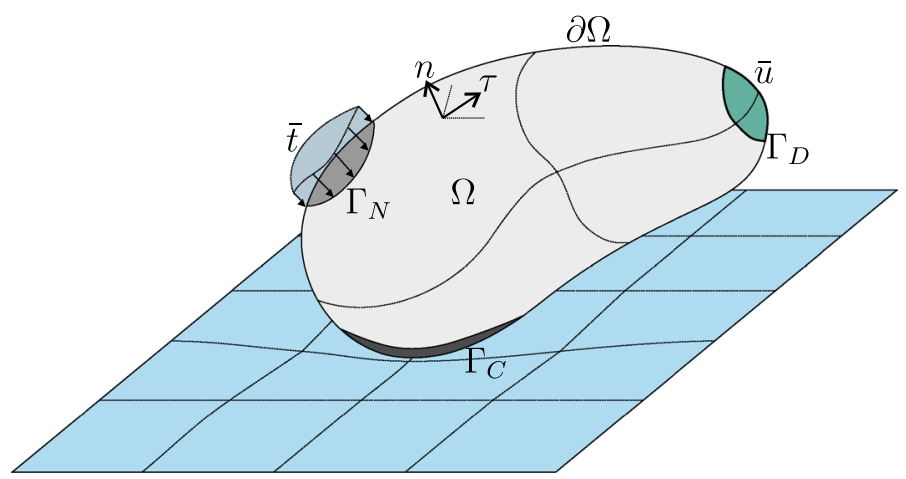

Fig. 1 Contact problem

consider the energy shape functional associated to the frictionless contact problem allowing a small interpenetration between the elastic body and a rigid foundation, developed in [16]. For the asymptotic analysis, we use a domain decomposition technique and an associated Steklov-Poincaré pseudodifferential operator. The differentiability of the energy of this new class of variational problems, with respect to the nonsmooth perturbation, is established. A closed form for the topological derivative in the three-dimensional space is also presented.

The paper is organized as follows. The frictionless contact problem with small interpenetration is presented in Sect. 2. The topological asymptotic analysis, with respect to the nucleation of spherical holes (voids) in three spatial dimensions, is developed with all details in Sect. 3; here, a closed form of the topological derivatives associated with the energy shape functional is presented. The paper ends with some concluding remarks in Sect. 4.

\section{Static Contact Model for Small Interpenetration}

We consider the problem of an elastic body having contact with a rigid foundation. The domain of the body, denoted by $\Omega \subset \mathbb{R}^{3}$, is assumed to be bounded and to have Lipschitz boundary $\partial \Omega$ consisting of three mutually disjoint parts $\Gamma_{D}, \Gamma_{N}$, and $\Gamma_{C}$ with positive measures, where different boundary conditions are prescribed. On $\Gamma_{D}$, we prescribe Dirichlet boundary conditions (displacement), on $\Gamma_{N}$ Neumann boundary conditions (traction), and on $\Gamma_{C}$ a contact condition with the rigid foundation that admits interpenetration; see Fig. 1.

For the contact on $\Gamma_{C}$, we consider only the normal compliance law of the type

$$
\sigma_{n}(\boldsymbol{u})=-p\left(u_{n}-g\right)
$$

where $u_{n}:=\boldsymbol{u} \cdot \boldsymbol{n}$ denotes the normal component of the displacement field $\boldsymbol{u}, \boldsymbol{n}$ is the unit outward normal vector to the boundary $\partial \Omega$, and $g$ is the gap on the potential contact zone. Moreover, in $(1), \sigma_{n}(\boldsymbol{u})$ represents the normal component to the boundary of the stress tensor $\sigma(\boldsymbol{u})$, i.e., $\sigma_{n}(\boldsymbol{u})=\boldsymbol{\sigma}(\boldsymbol{u}) \boldsymbol{n} \cdot \boldsymbol{n}$. The Cauchy stress tensor $\boldsymbol{\sigma}(\boldsymbol{u})$ is 
defined as

$$
\sigma(\boldsymbol{u}):=\mathbb{C} \varepsilon(\boldsymbol{u})
$$

where $\boldsymbol{\varepsilon}(\boldsymbol{u})$ is the symmetric part of the gradient of the displacement field $\boldsymbol{u}$, i.e.,

$$
\boldsymbol{\varepsilon}(\boldsymbol{u}):=\frac{1}{2}\left(\nabla \boldsymbol{u}+(\nabla \boldsymbol{u})^{\top}\right)
$$

and $\mathbb{C}$ denotes the fourth-order elastic tensor. For an isotropic elastic body, this tensor is given by

$$
\mathbb{C}=2 \mu \mathbb{I}+\lambda(\boldsymbol{I} \otimes \boldsymbol{I}),
$$

with $\mu$ and $\lambda$ denoting the Lamé coefficients. In the above expression, we use $\mathbb{I}$ and $\boldsymbol{I}$ to denote the fourth-order and the second-order identity tensors, respectively. In terms of the engineering constant $E$ (Young's modulus) and $v$ (Poisson's ratio), the above constitutive response can be written as

$$
\mathbb{C}=\frac{E}{1-v^{2}}[(1-v) \mathbb{I}+v(\boldsymbol{I} \otimes \boldsymbol{I})]
$$

The function $p: \mathbb{R} \rightarrow \overline{\mathbb{R}}_{+}=[0,+\infty]$ in (1) is used to model the interpenetration between the body and the foundation. There are two parameters, $\beta>\alpha$, where $\alpha$ indicates the initial contact and the value of $\beta$ describes a limit such that no further interpenetration is possible. The function $p$ is monotone with the following properties:

$$
\begin{aligned}
& p(y)=0 \quad \text { for } y \leq \alpha \text {, } \\
& \lim _{y \uparrow \beta} p(y)=+\infty \text {, } \\
& p(y)=+\infty \quad \text { for } y \geq \beta \text {. }
\end{aligned}
$$

The strong form of the equilibrium problem under this contact condition is to find a displacement field $\boldsymbol{u}: \Omega \rightarrow \mathbb{R}^{3}$ such that

$$
\begin{aligned}
& -\operatorname{div} \sigma(\boldsymbol{u})=\mathbf{0} \quad \text { in } \Omega, \\
& \boldsymbol{u}=\overline{\boldsymbol{u}} \quad \text { on } \Gamma_{D} \text {, } \\
& \boldsymbol{\sigma}(\boldsymbol{u}) \boldsymbol{n}=\overline{\boldsymbol{t}} \quad \text { on } \Gamma_{N}, \\
& \sigma_{n}(\boldsymbol{u}) \quad=-p\left(u_{n}-g\right) \text { on } \Gamma_{C} \text {, } \\
& \sigma_{\tau}(\boldsymbol{u})=\mathbf{0} \text { on } \Gamma_{C} \text {. }
\end{aligned}
$$

The last condition in (4) indicates that the contact is without friction, where $\sigma_{\tau}(\boldsymbol{u})=$ $\boldsymbol{\sigma}(\boldsymbol{u}) \boldsymbol{n}-\sigma_{n}(\boldsymbol{u}) \boldsymbol{n}$ denotes the tangential component of the stress tensor $\boldsymbol{\sigma}(\boldsymbol{u})$.

We assume that the stress operator $\sigma$ is bounded and positive definite, i.e., there exist two constants $\underline{\sigma}, \bar{\sigma}>0$ such that

$$
|\sigma| \leq \bar{\sigma}, \quad \forall \boldsymbol{\phi} \in \mathbb{R}^{3 \times 3}: \sigma(\phi) \cdot \boldsymbol{\phi} \geq \underline{\sigma}|\boldsymbol{\phi}|^{2}
$$




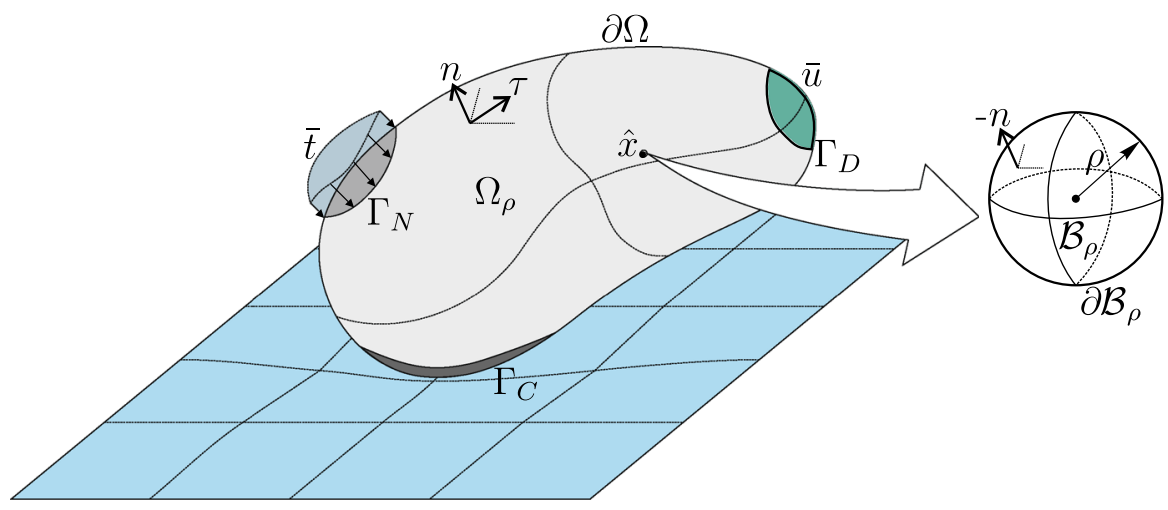

Fig. 2 Perturbed contact problem

and the data satisfy

$$
g \in H^{1 / 2}\left(\Gamma_{C}\right), \overline{\boldsymbol{u}} \in H^{1}\left(\Omega ; \mathbb{R}^{3}\right),\left.\bar{u}_{n}\right|_{\Gamma_{C}}=g \text { and } \overline{\boldsymbol{t}} \in\left(H^{1 / 2}\left(\Gamma_{N} ; \mathbb{R}^{3}\right)\right)^{*}
$$

The weak formulation of the problem stated in (4) is given by the following variational problem: find $\boldsymbol{u} \in \mathcal{U}$ with $u_{n}-g \in \operatorname{dom}(p)$ such that

$$
\langle\boldsymbol{\sigma}(\boldsymbol{u}), \boldsymbol{\varepsilon}(\boldsymbol{v})-\boldsymbol{\varepsilon}(\boldsymbol{u})\rangle_{\Omega}+\left\langle p\left(u_{n}-g\right), v_{n}-u_{n}\right\rangle_{\Gamma_{C}}=\langle\overline{\boldsymbol{t}}, \boldsymbol{v}-\boldsymbol{u}\rangle_{\Gamma_{N}} \quad \forall \boldsymbol{v} \in \mathcal{U},
$$

where the set $\mathcal{U}$ of admissible functions is given by

$$
\mathcal{U}:=\left\{\varphi \in H^{1}\left(\Omega ; \mathbb{R}^{3}\right): \varphi=\overline{\boldsymbol{u}} \text { on } \Gamma_{D}\right\}
$$

and the domain of definition of the function $p$ is

$$
\begin{aligned}
& \operatorname{dom}(p):= \\
& \qquad \varphi \in L^{1}\left(\Gamma_{C}\right): p(\varphi) \in L^{1}\left(\Gamma_{C}\right), \exists C>0: \int_{\Gamma_{C}} p(\varphi) v \leq C\|v\|_{H^{1 / 2}\left(\Gamma_{C}\right)} .
\end{aligned}
$$

For a complete and detailed description of this model, we refer the reader to [16], where it was proved that, under the above assumptions, problem (5) has a unique solution.

\section{Topological Asymptotic Analysis}

In this section, we obtain an asymptotic expansion for the energy shape functional when a small spheroidal cavity of radius $\rho$ is introduced at an arbitrary point $\hat{\boldsymbol{x}}$ of $\Omega$, far enough from the potential contact region $\Gamma_{C}$; see Fig. 2. 
The main term of this expansion is the topological derivative operator, which represents a first-order asymptotic correction term of the given shape functional with respect to the singular domain perturbation [4].

Let us consider a shape functional defined on $\Omega$ and depending on the solution $\boldsymbol{u}$, denoted by $\mathcal{J}_{\Omega}(\boldsymbol{u})$. After the introduction of a singular perturbation at $\hat{\boldsymbol{x}}$, we have a new domain $\Omega_{\rho}:=\Omega \backslash \overline{\mathcal{B}_{\rho}}$, where $\mathcal{B}_{\rho}$ is the ball of radius $\rho$ centered at $\hat{\boldsymbol{x}}$, that is, $\mathcal{B}_{\rho}:=\left\{\boldsymbol{x} \in \mathbb{R}^{3}:|\boldsymbol{x}-\hat{\boldsymbol{x}}|<\rho\right\}$; see Fig. 2 .

Therefore, an asymptotic expansion of the energy shape functional defined on the perturbed domain $\Omega_{\rho}$, i.e., $\mathcal{J}_{\Omega_{\rho}}$, can be written as

$$
\mathcal{J}_{\Omega_{\rho}}\left(\boldsymbol{u}_{\rho}\right)=\mathcal{J}_{\Omega}(\boldsymbol{u})+f(\rho) \mathcal{T}_{\Omega}(\hat{\boldsymbol{x}})+o(f(\rho))
$$

where $f(\rho)$ is a decreasing positive function such that $f(\rho) \rightarrow 0$ as $\rho \downarrow 0, \mathcal{T}_{\Omega}(\hat{\boldsymbol{x}})$ is the topological derivative of $\mathcal{J}_{\Omega}$ at $\hat{\boldsymbol{x}}$, and $\boldsymbol{u}_{\rho}$ is the solution of the following contact problem in the perturbed domain: find a displacement field $\boldsymbol{u}_{\rho}: \Omega_{\rho} \rightarrow \mathbb{R}^{3}$ such that

$$
\begin{aligned}
& -\operatorname{div} \sigma\left(\boldsymbol{u}_{\rho}\right)=\mathbf{0} \quad \text { in } \Omega_{\rho},
\end{aligned}
$$

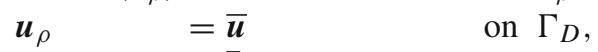

$$
\begin{aligned}
& \boldsymbol{\sigma}\left(\boldsymbol{u}_{\rho}\right) \boldsymbol{n}=\overline{\boldsymbol{t}} \text { on } \Gamma_{N}, \\
& \sigma_{n}\left(\boldsymbol{u}_{\rho}\right)=-p\left(\boldsymbol{u}_{\rho} \cdot \boldsymbol{n}-g\right) \text { on } \Gamma_{C} \text {, } \\
& \sigma_{\tau}\left(u_{\rho}\right)=\mathbf{0} \text { on } \Gamma_{C} \text {, } \\
& \boldsymbol{\sigma}\left(\boldsymbol{u}_{\rho}\right) \boldsymbol{n}=\mathbf{0} \text { on } \partial \mathcal{B}_{\rho} \text {. }
\end{aligned}
$$

Note that there is no traction applied on the boundary of the hole, i.e., the homogeneous Neumann boundary condition is assumed on $\partial \mathcal{B}_{\rho}$.

From (8), in accordance with the classical definition of the topological derivative (see [4]), we have

$$
\mathcal{T}_{\Omega}(\hat{\boldsymbol{x}}):=\lim _{\rho \downarrow 0} \frac{\mathcal{J}_{\Omega_{\rho}}\left(\boldsymbol{u}_{\rho}\right)-\mathcal{J}_{\Omega}(\boldsymbol{u})}{f(\rho)} .
$$

In order to perform the asymptotic expansion and evaluation of the topological derivative of problem (9), in this work, we apply a domain decomposition method and the associated Steklov-Poincaré pseudodifferential operator.

\subsection{Domain Decomposition}

We start by decomposing the domain $\Omega_{\rho}$ into two parts: (i) a ball $\mathcal{B}_{R}$ of radius $R>$ $\rho>0$ centered at $\hat{\boldsymbol{x}} \in \Omega$, that is, $\mathcal{B}_{R}:=\left\{\boldsymbol{x} \in \mathbb{R}^{3}:|\boldsymbol{x}-\hat{\boldsymbol{x}}|<R\right\}$, and (ii) the domain $\Omega_{R}:=\Omega \backslash \overline{\mathcal{B}_{R}}$. Clearly, $\mathcal{B}_{R}$ contains the small cavity $\mathcal{B}_{\rho}$, and, for this perturbed configuration, we can define the domain as $C(R, \rho):=\mathcal{B}_{R} \backslash \overline{\mathcal{B}_{\rho}}$; see Fig. 3.

We use $\Gamma_{R}$ to denote the exterior boundary $\partial \mathcal{B}_{R}$ of the domain $C(R, \rho)$. 


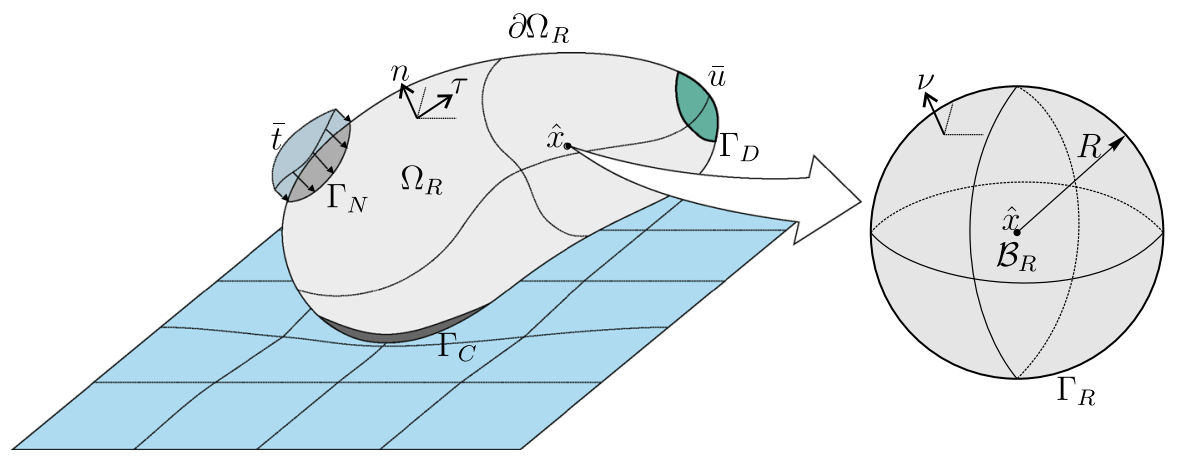

(a) Domains $\Omega_{R}$ and $\mathcal{B}_{R}$.

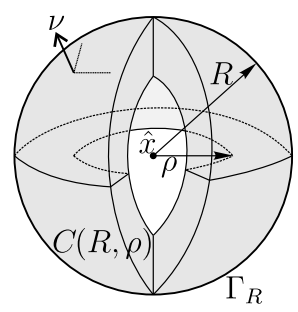

(b) Domain $C(R, \rho)$.

Fig. 3 Decomposition of the domain $\Omega$

First, we consider the following linear elasticity problem in $C(R, \rho)$ : given $\overline{\boldsymbol{v}} \in$ $H^{1 / 2}\left(\Gamma_{R} ; \mathbb{R}^{3}\right)$, find a displacement field $\omega_{\rho}: C(R, \rho) \rightarrow \mathbb{R}^{3}$ such that

$$
\begin{array}{ll}
-\operatorname{div} \sigma\left(\boldsymbol{\omega}_{\rho}\right) & =\mathbf{0} \text { in } C(R, \rho), \\
\boldsymbol{\omega}_{\rho} & =\overline{\boldsymbol{v}} \text { on } \Gamma_{R}, \\
\boldsymbol{\sigma}\left(\boldsymbol{\omega}_{\rho}\right) \boldsymbol{n} & =\mathbf{0} \text { on } \partial \mathcal{B}_{\rho} .
\end{array}
$$

Using (11), we can define the Steklov-Poincaré boundary operator $\mathcal{S}_{\rho}$ on $\Gamma_{R}$ as

$$
\mathcal{S}_{\rho}: \bar{v} \in H^{1 / 2}\left(\Gamma_{R} ; \mathbb{R}^{3}\right) \rightarrow \sigma\left(\omega_{\rho}\right) v \in H^{-1 / 2}\left(\Gamma_{R} ; \mathbb{R}^{3}\right)
$$

where $\boldsymbol{v}$ denotes the unit normal vector to the boundary $\Gamma_{R}$ pointing outside the ball $\mathcal{B}_{R}$. Next, we consider the following contact problem in $\Omega_{R}$ : find a displacement field $\boldsymbol{u}_{\rho}^{R}: \Omega_{R} \rightarrow \mathbb{R}^{3}$ such that

$$
\begin{aligned}
& -\operatorname{div} \sigma\left(\boldsymbol{u}_{\rho}^{R}\right)=\mathbf{0} \quad \text { in } \Omega_{R}, \\
& \boldsymbol{u}_{\rho}^{R}=\overline{\boldsymbol{u}} \quad \text { on } \Gamma_{D} \text {, } \\
& \boldsymbol{\sigma}\left(\boldsymbol{u}_{\rho}^{R}\right) \boldsymbol{n}=\overline{\boldsymbol{t}} \quad \text { on } \Gamma_{N}, \\
& \sigma_{n}\left(\boldsymbol{u}_{\rho}^{R}\right)=-p\left(\boldsymbol{u}_{\rho}^{R} \cdot \boldsymbol{n}-g\right) \text { on } \Gamma_{C} \text {, } \\
& \sigma_{\tau}\left(\boldsymbol{u}_{\rho}^{R}\right)=\mathbf{0} \quad \text { on } \Gamma_{C}, \\
& \boldsymbol{\sigma}\left(\boldsymbol{u}_{\rho}^{R}\right) \boldsymbol{v} \quad=\mathcal{S}_{\rho}\left(\boldsymbol{u}_{\rho}^{R}\right) \quad \text { on } \Gamma_{R} \text {. }
\end{aligned}
$$


Its variational formulation can be written as follows: find a displacement field $\boldsymbol{u}_{\rho}^{R} \in \mathcal{U}_{R}$ with $p\left(\boldsymbol{u}_{\rho}^{R} \cdot \boldsymbol{n}-g\right) \in \operatorname{dom}(p)$ such that

$$
\begin{array}{r}
\left\langle\boldsymbol{\sigma}\left(\boldsymbol{u}_{\rho}^{R}\right), \boldsymbol{\varepsilon}(\boldsymbol{v})-\boldsymbol{\varepsilon}\left(\boldsymbol{u}_{\rho}^{R}\right)\right\rangle_{\Omega_{R}}+\left\langle p\left(\boldsymbol{u}_{\rho}^{R} \cdot \boldsymbol{n}-g\right), v_{n}-\boldsymbol{u}_{\rho}^{R} \cdot \boldsymbol{n}\right\rangle_{\Gamma_{C}} \\
+\left\langle\mathcal{S}_{\rho}\left(\boldsymbol{u}_{\rho}^{R}\right), \boldsymbol{v}-\boldsymbol{u}_{\rho}^{R}\right\rangle_{\Gamma_{R}}=\left\langle\overline{\boldsymbol{t}}, \boldsymbol{v}-\boldsymbol{u}_{\rho}^{R}\right\rangle_{\Gamma_{N}} \quad \forall \boldsymbol{v} \in \mathcal{U}_{R},
\end{array}
$$

where the set $\mathcal{U}_{R}$ of admissible functions is given by

$$
\mathcal{U}_{R}:=\left\{\varphi \in H^{1}\left(\Omega_{R} ; \mathbb{R}^{3}\right): \varphi=\overline{\boldsymbol{u}} \text { on } \Gamma_{D}\right\}
$$

From (11) and (12), it follows that the solution $\boldsymbol{u}_{\rho}$ of (9) satisfies

$$
\boldsymbol{\sigma}\left(\boldsymbol{u}_{\rho}\right) \boldsymbol{v}=\mathcal{S}_{\rho}\left(\boldsymbol{u}_{\rho}\right) \text { on } \Gamma_{R}
$$

Consequently, the restriction of $\boldsymbol{u}_{\rho}$ to the truncated domain $\Omega_{R}$ coincides with the solution $\boldsymbol{u}_{\rho}^{R}$ of (13) and similarly $\left.\boldsymbol{u}_{\rho}\right|_{C(R, \rho)}=\omega_{\rho}$, where $\omega_{\rho}$ is the solution to (11) with $\overline{\boldsymbol{v}}=\left.\boldsymbol{u}_{\rho}\right|_{\Gamma_{R}}$. We also observe that, by the definition of the Steklov-Poincaré boundary operator in $C(R, \rho)$, the solution $\omega_{\rho}$ of (11) satisfies

$$
\int_{C(R, \rho)} \boldsymbol{\sigma}\left(\omega_{\rho}\right) \cdot \boldsymbol{\varepsilon}\left(\omega_{\rho}\right)=\left\langle\mathcal{S}_{\rho}(\overline{\boldsymbol{v}}), \overline{\boldsymbol{v}}\right\rangle_{\Gamma_{R}} .
$$

For the unperturbed case $(\rho=0)$, we define the Steklov-Poincaré operator as

$$
\mathcal{S}:=\mathcal{S}_{0}: \overline{\boldsymbol{v}} \in H^{1 / 2}\left(\Gamma_{R} ; \mathbb{R}^{3}\right) \rightarrow \boldsymbol{\sigma}(\omega) \boldsymbol{v} \in H^{-1 / 2}\left(\Gamma_{R} ; \mathbb{R}^{3}\right)
$$

associated with the problem

$$
\begin{array}{ll}
-\operatorname{div} \sigma(\boldsymbol{\omega}) & =\mathbf{0} \text { in } \mathcal{B}_{R}, \\
\boldsymbol{\omega} & =\overline{\boldsymbol{v}} \text { on } \Gamma_{R} .
\end{array}
$$

Applying the domain decomposition technique to the problem (4) on $\Omega$, we can rewrite (5) as follows:

$$
\begin{array}{r}
\langle\boldsymbol{\sigma}(\boldsymbol{u}), \boldsymbol{\varepsilon}(\boldsymbol{v})-\boldsymbol{\varepsilon}(\boldsymbol{u})\rangle_{\Omega_{R}}+\left\langle p\left(u_{n}-g\right), v_{n}-u_{n}\right\rangle_{\Gamma_{C}}+\langle\mathcal{S}(\boldsymbol{u}), \boldsymbol{v}-\boldsymbol{u}\rangle_{\Gamma_{R}} \\
=\langle\overline{\boldsymbol{t}}, \boldsymbol{v}-\boldsymbol{u}\rangle_{\Gamma_{N}} \quad \forall \boldsymbol{v} \in \mathcal{U}_{R} .
\end{array}
$$

It is well known that $\mathcal{S}_{\rho}$ is a positive definite operator for any $\rho \geq 0$, and that the following asymptotic expansion holds:

$$
\mathcal{S}_{\rho}=\mathcal{S}+\rho^{3} \mathcal{S}^{\prime}+o\left(\rho^{3}\right), \quad \rho \downarrow 0,
$$

with a bounded linear operator $\mathcal{S}^{\prime}[17]$. 


\subsection{Topological Derivative}

For the contact model studied in this work, the energy shape functional associated to the domain $\Omega$ is given by [16]

$$
\mathcal{J}_{\Omega}(\boldsymbol{u}):=\frac{1}{2}\langle\boldsymbol{\sigma}(\boldsymbol{u}), \boldsymbol{\varepsilon}(\boldsymbol{u})\rangle_{\Omega}-\langle\overline{\boldsymbol{t}}, \boldsymbol{u}\rangle_{\Gamma_{N}}+\int_{\Gamma_{C}} P\left(u_{n}-g\right)
$$

where $\boldsymbol{u}$ denotes the solution of the problem in the unperturbed domain (see (4)), and the function $P(y)$ is given by

$$
P(y):=\int_{-\infty}^{y} p(z)
$$

Considering the singular perturbation $\mathcal{B}_{\rho}$, the energy shape functional associated to the perturbed domain $\Omega_{\rho}$ is given by

$$
\mathcal{J}_{\Omega_{\rho}}\left(\boldsymbol{u}_{\rho}\right):=\frac{1}{2}\left\langle\boldsymbol{\sigma}\left(\boldsymbol{u}_{\rho}\right), \boldsymbol{\varepsilon}\left(\boldsymbol{u}_{\rho}\right)\right\rangle_{\Omega_{\rho}}-\left\langle\overline{\boldsymbol{t}}, \boldsymbol{u}_{\rho}\right\rangle_{\Gamma_{N}}+\int_{\Gamma_{C}} P\left(\boldsymbol{u}_{\rho} \cdot \boldsymbol{n}-g\right)
$$

where $\boldsymbol{u}_{\rho}$ is the solution of the problem in $\Omega_{\rho}$ [(see (9)].

Now, by taking into account the domain decomposition and the Steklov-Poincaré boundary operator presented above, we can define the following functional associated to the truncated domain $\Omega_{R}$ :

$$
\begin{gathered}
\mathcal{I}_{\Omega_{R}}\left(\boldsymbol{u}_{\rho}^{R}\right):=\frac{1}{2}\left\langle\boldsymbol{\sigma}\left(\boldsymbol{u}_{\rho}^{R}\right), \boldsymbol{\varepsilon}\left(\boldsymbol{u}_{\rho}^{R}\right)\right\rangle_{\Omega_{R}}-\left\langle\overline{\boldsymbol{t}}, \boldsymbol{u}_{\rho}^{R}\right\rangle_{\Gamma_{N}}+\int_{\Gamma_{C}} P\left(\boldsymbol{u}_{\rho}^{R} \cdot \boldsymbol{n}-g\right) \\
+\frac{1}{2}\left\langle\mathcal{S}_{\rho}\left(\boldsymbol{u}_{\rho}^{R}\right), \boldsymbol{u}_{\rho}^{R}\right\rangle_{\Gamma_{R}} .
\end{gathered}
$$

In terms of the above functional, the contact problem in the truncated domain $\Omega_{R}$, given by (13), can be written as the following optimization problem: the displacement field $\boldsymbol{u}_{\rho}^{R}$ is the unique minimizer such that

$$
\mathcal{I}_{\Omega_{R}}\left(\boldsymbol{u}_{\rho}^{R}\right)=\inf _{\boldsymbol{v} \in \operatorname{dom}\left(\mathcal{I}_{\Omega_{R}}\right)} \mathcal{I}_{\Omega_{R}}(\boldsymbol{v})
$$

where

$$
\operatorname{dom}\left(\mathcal{I}_{\Omega_{R}}\right):=\left\{v \in \mathcal{U}_{R}: P\left(v_{n}-g\right) \in L^{1}\left(\Gamma_{C}\right)\right\} .
$$

For the optimization problem (24), we can establish the equivalence

$$
\mathcal{I}_{\Omega_{R}}\left(\boldsymbol{u}_{\rho}^{R}\right) \equiv \mathcal{J}_{\Omega_{\rho}}\left(\boldsymbol{u}_{\rho}\right)
$$


since the minimizer in (24) coincides with the restriction to $\Omega_{R}$ of the minimizer $\boldsymbol{u}_{\rho}$ of the corresponding quadratic functional defined in the whole singularly perturbed domain $\Omega_{\rho}$.

Proposition 3.1 Let $\boldsymbol{u}$ and $\boldsymbol{u}_{\rho}$ be the solutions to (5) and (14), respectively. Then

$$
\boldsymbol{u}_{\rho} \rightarrow \boldsymbol{u} \text { strongly in } H^{1}\left(\Omega_{R} ; \mathbb{R}^{3}\right) \text { as } \rho \downarrow 0 \text {. }
$$

Proof First, we show that the sequence $\left\{\boldsymbol{u}_{\rho}\right\}, \rho \downarrow 0$, is bounded in $H^{1}\left(\Omega_{R} ; \mathbb{R}^{3}\right)$. Using $\boldsymbol{v}:=2 \boldsymbol{u}_{\rho}-\overline{\boldsymbol{u}}$ as a test function in (14), we obtain

$$
\begin{array}{r}
\left\langle\boldsymbol{\sigma}\left(\boldsymbol{u}_{\rho}\right), \boldsymbol{\varepsilon}\left(\boldsymbol{u}_{\rho}\right)\right\rangle_{\Omega_{R}}+\left\langle p\left(\boldsymbol{u}_{\rho} \cdot \boldsymbol{n}-g\right), \boldsymbol{u}_{\rho} \cdot \boldsymbol{n}-g\right\rangle_{\Gamma_{C}}+\left\langle\mathcal{S}_{\rho}\left(\boldsymbol{u}_{\rho}\right), \boldsymbol{u}_{\rho}\right\rangle \\
=\left\langle\boldsymbol{\sigma}\left(\boldsymbol{u}_{\rho}\right), \boldsymbol{\varepsilon}(\overline{\boldsymbol{u}})\right\rangle_{\Omega_{R}}+\left\langle p\left(\boldsymbol{u}_{\rho} \cdot \boldsymbol{n}-g\right), \bar{u}_{n}-g\right\rangle_{\Gamma_{C}} \\
+\left\langle\mathcal{S}_{\rho}\left(\boldsymbol{u}_{\rho}\right), \overline{\boldsymbol{u}}\right\rangle_{\Gamma_{R}}+\left\langle\overline{\boldsymbol{t}}, \boldsymbol{u}_{\rho}-\overline{\boldsymbol{u}}\right\rangle_{\Gamma_{N}} .
\end{array}
$$

The terms on the right-hand side can be estimated using the boundedness of $\sigma$, the expression (16), and the properties of the data $\overline{\boldsymbol{u}}$ and $\overline{\boldsymbol{t}}$ as follows:

$$
\begin{aligned}
\left\langle\boldsymbol{\sigma}\left(\boldsymbol{u}_{\rho}\right), \boldsymbol{\varepsilon}(\overline{\boldsymbol{u}})\right\rangle_{\Omega_{R}}+\left\langle\mathcal{S}_{\rho}\left(\boldsymbol{u}_{\rho}\right), \overline{\boldsymbol{u}}\right\rangle_{\Gamma_{R}} & =\left\langle\boldsymbol{\sigma}\left(\boldsymbol{u}_{\rho}\right), \boldsymbol{\varepsilon}(\overline{\boldsymbol{u}})\right\rangle_{\Omega_{\rho}} \\
\leq & \bar{\sigma}\left\|\boldsymbol{\varepsilon}\left(\boldsymbol{u}_{\rho}\right)\right\|_{L^{2}\left(\Omega_{\rho}, \mathbb{R}^{3}\right)}\|\boldsymbol{\varepsilon}(\overline{\boldsymbol{u}})\|_{L^{2}\left(\Omega_{\rho}, \mathbb{R}^{3}\right)}, \\
\left\langle p\left(\boldsymbol{u}_{\rho} \cdot \boldsymbol{n}-g\right), \bar{u}_{n}-g\right\rangle_{\Gamma_{C}}= & 0, \\
\left\langle\overline{\boldsymbol{t}}, \boldsymbol{u}_{\rho}-\overline{\boldsymbol{u}}\right\rangle_{\Gamma_{N}} \leq & \|\overline{\boldsymbol{t}}\|_{\left(H^{1 / 2}\left(\Gamma_{N} ; \mathbb{R}^{3}\right)\right)}\left(\left\|\boldsymbol{u}_{\rho}\right\|_{H^{1 / 2}\left(\Gamma_{N} ; \mathbb{R}^{3}\right)}\right. \\
& \left.+\|\overline{\boldsymbol{u}}\|_{H^{1 / 2}\left(\Gamma_{N} ; \mathbb{R}^{3}\right)}\right) .
\end{aligned}
$$

Using positive definiteness of $\sigma$, the expression (16), and the monotonicity of $p$, we get a lower bound for the left-hand side of (28):

$$
\begin{array}{r}
\left\langle\boldsymbol{\sigma}\left(\boldsymbol{u}_{\rho}\right), \boldsymbol{\varepsilon}\left(\boldsymbol{u}_{\rho}\right)\right\rangle_{\Omega_{R}}+\left\langle p\left(\boldsymbol{u}_{\rho} \cdot \boldsymbol{n}-g\right), \boldsymbol{u}_{\rho} \cdot \boldsymbol{n}-g\right\rangle_{\Gamma_{C}}+\left\langle\mathcal{S}_{\rho}\left(\boldsymbol{u}_{\rho}\right), \boldsymbol{u}_{\rho}\right\rangle_{\Gamma_{R}} \\
\geq \underline{\sigma}\left\|\boldsymbol{\varepsilon}\left(\boldsymbol{u}_{\rho}\right)\right\|_{L^{2}\left(\Omega_{\rho} ; \mathbb{R}^{3}\right)}^{2}
\end{array}
$$

Combining the above estimates with (28), we find that there is a constant $C_{1}>0$ depending only on $\underline{\sigma}, \bar{\sigma},\|\overline{\boldsymbol{u}}\|_{H^{1}\left(\Omega ; \mathbb{R}^{3}\right)}$ and $\|\overline{\boldsymbol{t}}\|_{\left(H^{1 / 2}\left(\Gamma_{N} ; \mathbb{R}^{3}\right)\right)^{*}}$ such that

$$
\left\|\boldsymbol{\varepsilon}\left(\boldsymbol{u}_{\rho}\right)\right\|_{L^{2}\left(\Omega_{\rho} ; \mathbb{R}^{3}\right)}^{2} \leq C_{1}\left(\left\|\boldsymbol{\varepsilon}\left(\boldsymbol{u}_{\rho}\right)\right\|_{L^{2}\left(\Omega_{\rho} ; \mathbb{R}^{3}\right)}+\left\|\boldsymbol{u}_{\rho}\right\|_{H^{1 / 2}\left(\Gamma_{N} ; \mathbb{R}^{3}\right)}+\|\overline{\boldsymbol{u}}\|_{H^{1 / 2}\left(\Gamma_{N} ; \mathbb{R}^{3}\right)}\right) .
$$

Now, we use the embedding $H^{1}\left(\Omega_{R} ; \mathbb{R}^{3}\right) \hookrightarrow H^{1 / 2}\left(\Gamma_{N} ; \mathbb{R}^{3}\right)$, Young's inequality, and Korn's inequality in $H^{1}\left(\Omega_{R} ; \mathbb{R}^{3}\right)$ to obtain

$$
\left\|\boldsymbol{u}_{\rho}\right\|_{H^{1}\left(\Omega_{R} ; \mathbb{R}^{3}\right)} \leq C_{K}\left\|\boldsymbol{\varepsilon}\left(\boldsymbol{u}_{\rho}\right)\right\|_{L^{2}\left(\Omega_{R} ; \mathbb{R}^{3}\right)} \leq C_{K}\left\|\boldsymbol{\varepsilon}\left(\boldsymbol{u}_{\rho}\right)\right\|_{L^{2}\left(\Omega_{\rho}, \mathbb{R}^{3}\right)} \leq C_{2},
$$


where $C_{K}>0$ is the constant in the Korn inequality and $C_{2}>0$ depends on the same quantities as $C_{1}$. To show strong convergence, we test (14) with $\boldsymbol{v}:=\boldsymbol{u}$ and (18) with $\boldsymbol{v}:=\boldsymbol{u}_{\rho}$. Adding the resulting equations and multiplying by -1 , we obtain

$$
\begin{array}{r}
\left\langle\boldsymbol{\sigma}\left(\boldsymbol{u}_{\rho}\right)-\boldsymbol{\sigma}(\boldsymbol{u}), \boldsymbol{\varepsilon}\left(\boldsymbol{u}_{\rho}\right)-\boldsymbol{\varepsilon}(\boldsymbol{u})\right\rangle_{\Omega_{R}}+\left\langle p\left(\boldsymbol{u}_{\rho} \cdot \boldsymbol{n}-g\right)-p\left(u_{n}-g\right), \boldsymbol{u}_{\rho} \cdot \boldsymbol{n}-u_{n}\right\rangle_{\Gamma_{C}} \\
+\left\langle\mathcal{S}_{\rho}\left(\boldsymbol{u}_{\rho}\right)-\mathcal{S}(\boldsymbol{u}), \boldsymbol{u}_{\rho}-\boldsymbol{u}\right\rangle_{\Gamma_{R}}=0 .
\end{array}
$$

Since $\mathcal{S}_{\rho}$ is a positive definite operator which admits the asymptotic expansion (19), and the sequence $\left\{\boldsymbol{u}_{\rho}\right\}$ is bounded in $H^{1}\left(\Omega_{R} ; \mathbb{R}^{3}\right)$, the last term in (29) satisfies

$$
\begin{aligned}
\left\langle\mathcal{S}_{\rho}\left(\boldsymbol{u}_{\rho}\right)-\mathcal{S}(\boldsymbol{u}), \boldsymbol{u}_{\rho}-\boldsymbol{u}\right\rangle_{\Gamma_{R}}= & \left\langle\mathcal{S}_{\rho}\left(\boldsymbol{u}_{\rho}\right)-\mathcal{S}_{\rho}(\boldsymbol{u}), \boldsymbol{u}_{\rho}-\boldsymbol{u}\right\rangle_{\Gamma_{R}} \\
& +\left\langle\mathcal{S}_{\rho}(\boldsymbol{u})-\mathcal{S}(\boldsymbol{u}), \boldsymbol{u}_{\rho}-\boldsymbol{u}\right\rangle_{\Gamma_{R}} \\
\geq & \left\langle\rho^{3} \mathcal{S}^{\prime}(\boldsymbol{u}), \boldsymbol{u}_{\rho}-\boldsymbol{u}\right\rangle_{\Gamma_{R}}+o\left(\rho^{3}\right) \rightarrow 0 \text { as } \rho \downarrow 0 .
\end{aligned}
$$

Using this, together with the Korn inequality and the facts that $\sigma$ is positive definite and $p$ is nondecreasing, we deduce from (29) that

$$
\lim _{\rho \downarrow 0}\left\|\boldsymbol{u}_{\rho}-\boldsymbol{u}\right\|_{H^{1}\left(\Omega_{R} ; \mathbb{R}^{3}\right)}^{2} \leq 0
$$

as desired.

Proposition 3.2 The functional $\mathcal{I}_{\Omega_{R}}$, defined in (23), is right-differentiable at $\rho=0$ for any fixed $R>\rho$ with $\rho \geq 0$, and its derivative is

$$
\mathcal{I}_{\Omega_{R}}^{\prime}=\frac{1}{2} \int_{\Gamma_{R}} \mathcal{S}^{\prime}\left(\boldsymbol{u}^{R}\right) \boldsymbol{u}^{R}=\frac{1}{2}\left\langle\mathcal{S}^{\prime}\left(\boldsymbol{u}^{R}\right), \boldsymbol{u}^{R}\right\rangle_{\Gamma_{R}}
$$

where $\mathcal{S}^{\prime}$ is the main term of the asymptotic expansion of the Steklov-Poincaré boundary operator $\mathcal{S}_{\rho}$ in the space of Steklov-Poincaré operators, given by

$$
\mathcal{S}_{\rho}=\mathcal{S}+\rho^{3} \mathcal{S}^{\prime}+o\left(\rho^{3}\right)
$$

Proof The derivative of $\mathcal{I}_{\Omega_{R}}$ at $\rho=0$ can be written as

$$
\mathcal{I}_{\Omega_{R}}^{\prime}:=\lim _{\rho \downarrow 0} \frac{\mathcal{I}_{\Omega_{R}}\left(\boldsymbol{u}_{\rho}^{R}\right)-\mathcal{I}_{\Omega}\left(\boldsymbol{u}^{R}\right)}{\rho^{3}} .
$$

Let us consider the following inequalities:

$$
\frac{\mathcal{I}_{\Omega_{R}}\left(\boldsymbol{u}_{\rho}^{R}\right)-\mathcal{I}_{\Omega}\left(\boldsymbol{u}_{\rho}^{R}\right)}{\rho^{3}} \leq \mathcal{I}_{\Omega_{R}}^{\prime} \leq \frac{\mathcal{I}_{\Omega_{R}}\left(\boldsymbol{u}^{R}\right)-\mathcal{I}_{\Omega}\left(\boldsymbol{u}^{R}\right)}{\rho^{3}}
$$


Now, for the left-hand side of (33), we have

$$
\begin{gathered}
\frac{\mathcal{I}_{\Omega_{R}}\left(\boldsymbol{u}_{\rho}^{R}\right)-\mathcal{I}_{\Omega}\left(\boldsymbol{u}_{\rho}^{R}\right)}{\rho^{3}}=\frac{1}{\rho^{3}}\left\{\frac{1}{2}\left\langle\boldsymbol{\sigma}\left(\boldsymbol{u}_{\rho}^{R}\right), \boldsymbol{\varepsilon}\left(\boldsymbol{u}_{\rho}^{R}\right)\right\rangle_{\Omega_{R}}-\left\langle\overline{\boldsymbol{t}}, \boldsymbol{u}_{\rho}^{R}\right\rangle_{\Gamma_{N}}+\int_{\Gamma_{C}} P\left(\boldsymbol{u}_{\rho}^{R} \cdot \boldsymbol{n}-g\right)\right. \\
+\frac{1}{2}\left\langle\mathcal{S}_{\rho}\left(\boldsymbol{u}_{\rho}^{R}\right), \boldsymbol{u}_{\rho}^{R}\right\rangle_{\Gamma_{R}}-\frac{1}{2}\left\langle\boldsymbol{\sigma}\left(\boldsymbol{u}_{\rho}^{R}\right), \boldsymbol{\varepsilon}\left(\boldsymbol{u}_{\rho}^{R}\right)\right\rangle_{\Omega}+\left\langle\overline{\boldsymbol{t}}, \boldsymbol{u}_{\rho}^{R}\right\rangle_{\Gamma_{N}} \\
\left.-\int_{\Gamma_{C}} P\left(\boldsymbol{u}_{\rho}^{R} \cdot \boldsymbol{n}-g\right)\right\} \\
=\frac{1}{\rho^{3}}\left\{\frac{1}{2}\left\langle\boldsymbol{\sigma}\left(\boldsymbol{u}_{\rho}^{R}\right), \boldsymbol{\varepsilon}\left(\boldsymbol{u}_{\rho}^{R}\right)\right\rangle_{\Omega_{R}}-\left\langle\overline{\boldsymbol{t}}, \boldsymbol{u}_{\rho}^{R}\right\rangle_{\Gamma_{N}}+\int_{\Gamma_{C}} P\left(\boldsymbol{u}_{\rho}^{R} \cdot \boldsymbol{n}-g\right)\right. \\
+\frac{1}{2}\left\langle\mathcal{S}_{\rho}\left(\boldsymbol{u}_{\rho}^{R}\right), \boldsymbol{u}_{\rho}^{R}\right\rangle_{\Gamma_{R}}-\frac{1}{2}\left\langle\boldsymbol{\sigma}\left(\boldsymbol{u}_{\rho}^{R}\right), \boldsymbol{\varepsilon}\left(\boldsymbol{u}_{\rho}^{R}\right)\right\rangle_{\Omega^{R}}+\left\langle\overline{\boldsymbol{t}}, \boldsymbol{u}_{\rho}^{R}\right\rangle_{\Gamma_{N}} \\
\left.-\int_{\Gamma_{C}} P\left(\boldsymbol{u}_{\rho}^{R} \cdot \boldsymbol{n}-g\right)-\frac{1}{2}\left\langle\mathcal{S}\left(\boldsymbol{u}_{\rho}^{R}\right), \boldsymbol{u}_{\rho}^{R}\right\rangle_{\Gamma_{R}}\right\} \\
=\frac{1}{2 \rho^{3}}\left\langle\mathcal{S}_{\rho}\left(\boldsymbol{u}_{\rho}^{R}\right)-\mathcal{S}\left(\boldsymbol{u}_{\rho}^{R}\right), \boldsymbol{u}_{\rho}^{R}\right\rangle_{\Gamma_{R}} .
\end{gathered}
$$

Considering the asymptotic expansion of the Steklov-Poincaré operator, we have

$$
\begin{gathered}
\frac{\mathcal{I}_{\Omega_{R}}\left(\boldsymbol{u}_{\rho}^{R}\right)-\mathcal{I}_{\Omega}\left(\boldsymbol{u}_{\rho}^{R}\right)}{\rho^{3}}=\frac{1}{2 \rho^{3}} \\
\left\langle\mathcal{S}\left(\boldsymbol{u}_{\rho}^{R}\right)+\rho^{3} \mathcal{S}^{\prime}\left(\boldsymbol{u}_{\rho}^{R}\right)+o\left(\rho^{3}\right)-\mathcal{S}\left(\boldsymbol{u}_{\rho}^{R}\right), \boldsymbol{u}_{\rho}^{R}\right\rangle_{\Gamma_{R}} \\
=\frac{1}{2}\left\langle\mathcal{S}^{\prime}\left(\boldsymbol{u}_{\rho}^{R}\right), \boldsymbol{u}_{\rho}^{R}\right\rangle_{\Gamma_{R}}+\frac{1}{2}\left\langle\frac{o\left(\rho^{3}\right)}{\rho^{3}}, \boldsymbol{u}_{\rho}^{R}\right\rangle_{\Gamma_{R}}
\end{gathered}
$$

Using the strong convergence of $\boldsymbol{u}_{\rho}^{R}$ to $\boldsymbol{u}^{R}$ and the linearity of $\mathcal{S}^{\prime}$, we obtain

$$
\lim _{\rho \downarrow 0} \frac{\mathcal{I}_{\Omega_{R}}\left(\boldsymbol{u}_{\rho}^{R}\right)-\mathcal{I}_{\Omega}\left(\boldsymbol{u}_{\rho}^{R}\right)}{\rho^{3}}=\frac{1}{2}\left\langle\mathcal{S}^{\prime}\left(\boldsymbol{u}^{R}\right), \boldsymbol{u}^{R}\right\rangle_{\Gamma_{R}} .
$$

Now, the right-hand side of (33) can be written as

$$
\begin{aligned}
\frac{\mathcal{I}_{\Omega_{R}}\left(\boldsymbol{u}^{R}\right)-\mathcal{I}_{\Omega}\left(\boldsymbol{u}^{R}\right)}{\rho^{3}}= & \frac{1}{\rho^{3}}\left\{\frac{1}{2}\left\langle\boldsymbol{\sigma}\left(\boldsymbol{u}^{R}\right), \boldsymbol{\varepsilon}\left(\boldsymbol{u}^{R}\right)\right\rangle_{\Omega_{R}}-\left\langle\overline{\boldsymbol{t}}, \boldsymbol{u}^{R}\right\rangle_{\Gamma_{N}}+\int_{\Gamma_{C}} P\left(u_{n}^{R}-g\right)\right. \\
& +\frac{1}{2}\left\langle\mathcal{S}_{\rho}\left(\boldsymbol{u}^{R}\right), \boldsymbol{u}^{R}\right\rangle_{\Gamma_{R}}-\frac{1}{2}\left\langle\boldsymbol{\sigma}\left(\boldsymbol{u}^{R}\right), \boldsymbol{\varepsilon}\left(\boldsymbol{u}^{R}\right)\right\rangle_{\Omega}+\left\langle\overline{\boldsymbol{t}}, \boldsymbol{u}^{R}\right\rangle_{\Gamma_{N}} \\
\left.\quad-\int_{\Gamma_{C}} P\left(u_{n}^{R}-g\right)\right\} & \\
= & \frac{1}{\rho^{3}}\left\{\frac{1}{2}\left\langle\boldsymbol{\sigma}\left(\boldsymbol{u}^{R}\right), \boldsymbol{\varepsilon}\left(\boldsymbol{u}^{R}\right)\right\rangle_{\Omega_{R}}-\left\langle\overline{\boldsymbol{t}}, \boldsymbol{u}^{R}\right\rangle_{\Gamma_{N}}+\int_{\Gamma_{C}} P\left(u_{n}^{R}-g\right)\right. \\
& +\frac{1}{2}\left\langle\mathcal{S}_{\rho}\left(\boldsymbol{u}^{R}\right), \boldsymbol{u}^{R}\right\rangle_{\Gamma_{R}}-\frac{1}{2}\left\langle\boldsymbol{\sigma}\left(\boldsymbol{u}^{R}\right), \boldsymbol{\varepsilon}\left(\boldsymbol{u}^{R}\right)\right\rangle_{\Omega^{R}}+\left\langle\overline{\boldsymbol{t}}, \boldsymbol{u}^{R}\right\rangle_{\Gamma_{N}} \\
& \left.\quad-\int_{\Gamma_{C}} P\left(u_{n}^{R}-g\right)-\frac{1}{2}\left\langle\mathcal{S}\left(\boldsymbol{u}^{R}\right), \boldsymbol{u}^{R}\right\rangle_{\Gamma_{R}}\right\} \\
= & \frac{1}{2 \rho^{3}}\left\langle\mathcal{S}_{\rho}\left(\boldsymbol{u}^{R}\right)-\mathcal{S}\left(\boldsymbol{u}^{R}\right), \boldsymbol{u}^{R}\right\rangle_{\Gamma_{R}} .
\end{aligned}
$$


Considering the asymptotic expansion of the Steklov-Poincaré operator, we have

$$
\begin{gathered}
\frac{\mathcal{I}_{\Omega_{R}}\left(\boldsymbol{u}^{R}\right)-\mathcal{I}_{\Omega}\left(\boldsymbol{u}^{R}\right)}{\rho^{3}}=\frac{1}{2 \rho^{3}}\left\langle\mathcal{S}\left(\boldsymbol{u}^{R}\right)+\rho^{3} \mathcal{S}^{\prime}\left(\boldsymbol{u}^{R}\right)+o\left(\rho^{3}\right)-\mathcal{S}\left(\boldsymbol{u}^{R}\right), \boldsymbol{u}^{R}\right\rangle_{\Gamma_{R}} \\
=\frac{1}{2}\left\langle\mathcal{S}^{\prime}\left(\boldsymbol{u}^{R}\right), \boldsymbol{u}^{R}\right\rangle_{\Gamma_{R}}+\frac{1}{2}\left\langle\frac{o\left(\rho^{3}\right)}{\rho^{3}}, \boldsymbol{u}^{R}\right\rangle_{\Gamma_{R}}
\end{gathered}
$$

By taking the limit of the above expression as $\rho \downarrow 0$, we obtain

$$
\lim _{\rho \downarrow 0} \frac{\mathcal{I}_{\Omega_{R}}\left(\boldsymbol{u}^{R}\right)-\mathcal{I}_{\Omega}\left(\boldsymbol{u}^{R}\right)}{\rho^{3}}=\frac{1}{2}\left\langle\mathcal{S}^{\prime}\left(\boldsymbol{u}^{R}\right), \boldsymbol{u}^{R}\right\rangle_{\Gamma_{R}} .
$$

Finally, the expressions (36) and (39) imply (30).

Remark 3.1 Using Proposition 3.2, the asymptotic expansion of the functional $\mathcal{I}_{\Omega_{R}}$ can be written as

$$
\mathcal{I}_{\Omega_{R}}=\mathcal{I}_{\Omega}+\frac{\rho^{3}}{2}\left\langle\mathcal{S}^{\prime}\left(\boldsymbol{u}^{R}\right), \boldsymbol{u}^{R}\right\rangle_{\Gamma_{R}}+o\left(\rho^{3}\right),
$$

and in view of the asymptotic expansion (8), we finally see that the topological derivative satisfies the following identity:

$$
\mathcal{T}_{\Omega}(\hat{\boldsymbol{x}})=\frac{1}{2}\left\langle\mathcal{S}^{\prime}\left(\boldsymbol{u}^{R}\right), \boldsymbol{u}^{R}\right\rangle_{\Gamma_{R}} .
$$

Proposition 3.2 establishes the differentiability of the energy shape functional for this contact model with respect to the nonsmooth perturbation denoted by $\mathcal{B}_{\rho}$. This is an abstract result, whose closed form for the topological derivative $\mathcal{T}_{\Omega}(\hat{\boldsymbol{x}})$ is presented in the next section.

\subsection{Topological Derivative Evaluation}

By the main result of the previous section, the energy shape functional has an asymptotic expansion as $\rho \downarrow 0$; see (8) and (41). This means that the asymptotic behavior of the energy in $C(R, \rho)$ holds in the whole domain $\Omega$. Now, we only need to compute the topological derivative for the energy shape functional in $C(R, \rho)$, with its associated elastic problem (11). In order to evaluate that topological derivative, we can use the techniques available in the literature: see for instance [4,21-23]. Finally, an explicit and analytical formula for the topological derivative $\mathcal{T}_{\Omega}(\hat{\boldsymbol{x}})$ is given in the following result:

Theorem 3.1 The energy shape functional of an elastic solid, characterized by the constitutive Eq. (2), with a spherical cavity of radius $\rho$ with homogeneous Neumann boundary condition and centered at a point $\hat{\boldsymbol{x}} \in \Omega$, admits for $\rho \downarrow 0$ the following asymptotic expansion:

$$
\mathcal{J}_{\Omega_{\rho}}\left(\boldsymbol{u}_{\rho}\right)=\mathcal{J}_{\Omega}(\boldsymbol{u})+\rho^{3} \pi \mathbb{H} \boldsymbol{\sigma}(\boldsymbol{u}(\hat{\boldsymbol{x}})) \cdot \boldsymbol{\varepsilon}(\boldsymbol{u}(\hat{\boldsymbol{x}}))+o\left(\rho^{3}\right) \quad \forall \hat{\boldsymbol{x}} \in \Omega,
$$


where $\boldsymbol{u}(\hat{\boldsymbol{x}})$ is the solution of problem (4) evaluated at $\hat{\boldsymbol{x}}$, and $\mathbb{H}$ is a fourth-order tensor defined as

$$
\mathbb{H}:=\frac{1-v}{7-5 v}\left(10 \mathbb{I}-\frac{1-5 v}{1-2 v} \boldsymbol{I} \otimes \boldsymbol{I}\right),
$$

where $v$ is the Poisson ratio of the elastic medium, and $\boldsymbol{I}$ and $\mathbb{I}$ are the second-order and fourth-order identity tensors, respectively.

Proof The reader interested in the proof of this result may refer to [24-26].

Remark 3.2 The fourth-order tensor $\mathbb{H}$ in (42) can be interpreted as the polarization tensor associated to this problem. This concept is very useful, since the topological derivative formula can be written explicitly in terms of this tensor. The reader interested in this topic may refer to $[13,27,28]$.

\section{Conclusions}

Topological derivatives are used in shape optimization [1] to determine the location of small inclusions or voids. There are applications for the optimal design in structural mechanics, or for numerical solution of inverse problems. Therefore, the knowledge of analytical form of topological derivatives is important in numerical methods of shape optimization. In general, the topological derivatives cannot be determined directly for variational inequalities. The existence of topological derivatives for contact problems with friction, dynamic contact problems, or elastic-plastic problems is not known, and it is a difficult open problem. There are no asymptotic analysis tools which can be applied in the general case of nonlinear structural models in mechanics. The only known results on topological derivatives of shape functionals are derived for the static, frictionless contact problems with the unilateral conditions [1].

An analytical expression for the topological derivative of the energy shape functional associated to a new frictionless contact model [16] has been derived. The model allows for a small interpenetration between an elastic body and a rigid foundation. The results obtained can be extended to some nonsmooth domains, including the cracks with nonpenetration conditions. This issue is a subject of current research.

The asymptotic analysis is developed for the specific case of spherical voids introduced in an elastic body. Similar results can be derived for elastic inclusions [1]. The results are obtained by an application of a nonoverlapping domain decomposition technique with the associated Steklov-Poincaré pseudodifferential operators. In this way, the differentiability of the energy can be established. The final formula is a simple analytical expression, in terms of the solution of the state equation and the constitutive parameters, evaluated at each point of the unperturbed domain. Therefore, numerical results can be obtained for this class of models and for shape-topological optimization problems.

We point out that the small interpenetration condition, prescribed in the potential contact zone, does not contribute explicitly to the first-order topological derivative of the energy functional. This means that the formula for the topological derivative of the energy functional for the contact problems takes the same form as was obtained for the classical elasticity problem for an isotropic and homogeneous medium [1,2]. 
The contribution of the contact model in the topological derivative is through the displacement field, the solution of the contact problem with a nonlinear boundary condition (small interpenetration). This information can be potentially used in the topological design of mechanical components, under contact conditions, to achieve a specified behavior.

The simple formula for the topological derivative of the energy functional for contact problems can be used in numerical methods of optimum design which is the subject of current research in this domain.

Acknowledgments This research was partially supported by LabEx CARMIN-CIMPA SMV programme (France). The work of Jan Sokolowski was partially supported by the ANR-12- BS01-0007 Optiform. The work of J. Stebel was supported by the ESF grant Optimization with PDE Constraints, by the Czech Science Foundation (GAČR) Grant No. 201/09/0917 and RVO 67985840.

Open Access This article is distributed under the terms of the Creative Commons Attribution License which permits any use, distribution, and reproduction in any medium, provided the original author(s) and the source are credited.

\section{References}

1. Novotny, A.A., Sokołowski, J.: Topological Derivatives in Shape Optimization. Interaction of Mechanics and Mathematics. Springer, Heidelberg (2013)

2. Sokołowski, J., Żochowski, A.: Topological derivatives of shape functionals for elasticity systems. Mech. Struct. Mach. 29, 331-349 (2001)

3. Nazarov, S.A., Sokołowski, J.: Asymptotic analysis of shape functionals. J. Math. Pure Appl. 82(2), 125-196 (2003)

4. Sokołowski, J., Żochowski, A.: On the topological derivative in shape optimization. SIAM J. Control Optim. 37(4), 1251-1272 (1999)

5. Amstutz, S., Novotny, A.A., de Souza Neto, E.A.: Topological derivative-based topology optimization of structures subject to Drucker-Prager stress constraints. Comput. Meth. Appl. Mech. Eng. 233-236, 233-236 (2012)

6. Hintermüller, M., Laurain, A., Novotny, A.A.: Second-order topological expansion for electrical impedance tomography. Adv. Comput. Math. 36, 235-265 (2012)

7. Hintermüller, M., Laurain, A.: Multiphase image segmentation and modulation recovery based on shape and topological sensitivity. J. Mmath. Imaging Vis. 35, 1-22 (2009)

8. Giusti, S.M., Novotny, A.A., de Souza Neto, E.A., Feijóo, R.A.: Sensitivity of the macroscopic elasticity tensor to topological microstructural changes. J. Mech. Phys. Solids 57(3), 555-570 (2009)

9. Van Goethem, N., Novotny, A.A.: Crack nucleation sensitivity analysis. Math. Method Appl. Sci. 33(16), 1978-1994 (2010)

10. Novotny, A.A., Sokołowski, J., de Souza Neto, E.A.: Topological sensitivity analysis of a multi-scale constitutive model considering a cracked microstructure. Math. Method. Appl. Sci. 33(5), 676-686 (2010)

11. Khludnev, A.M., Sokołowski, J., Szulc, K.: Shape and topological sensitivity analysis in domains with cracks. Appl. Math. 55(6), 433-469 (2010)

12. Sokołowski, J., Żochowski, A.: Optimality conditions for simultaneous topology and shape optimization. SIAM J. Control Optim. 42(4), 1198-1221 (2003)

13. Cardone, G., Nazarov, S.A., Sokołowski, J.: Asymptotic analysis, polarization matrices, and topological derivatives for piezoelectric materials with small voids. SIAM J. Control Optim. 48(6), 3925-3961 (2010)

14. Iguernane, M., Nazarov, S.A., Roche, J.-R., Sokołowski, J., Szulc, K.: Topological derivatives for semilinear elliptic equations. Int. J. Appl. Math. Comput. Sci. 19(2), 191-205 (2009)

15. Frémiot, G., Horn, W., Laurain, A., Rao, M., Sokołowski, J.: On the analysis of boundary value problems in nonsmooth domains. Dissert. Math. 462, 149 pp. (2009). 
16. Eck, C., Jarušek, J., Stará, J.: Normal compliance contact models with finite interpenetration. Arch. Ration. Mech. Anal. 208(1), 25-57 (2013)

17. Sokołowski, J., Żochowski, A.: Modelling of topological derivatives for contact problems. Numer. Math. 102(1), 145-179 (2005)

18. Fulmanski, P., Lauraine, A., Scheid, J.F., Sokołowski, J.: A level set method in shape and topology optimization for variational inequalities. Inter. J. Appl. Math. Comput. Sci. 17(3), 413-430 (2007)

19. Jarušek, J., Krbec, M., Rao, M., Sokołowski, J.: Conical differentiability for evolution variational inequalities. J. Differ. Equ. 193(1), 131-146 (2003)

20. Argatov, I.I., Sokołowski, J.: Asymptotics of the energy functional in the Signorini problem under small singular perturbation of the domain. Comp. Math. Math. Phys. 43(5), 710-724 (2003)

21. Amstutz, S.: Aspects Théoriques et Numériques en Optimisation de Forme Topologique. Phd thesis, Institut National des Sciences Appliquées de Toulouse, Toulouse (2003).

22. Novotny, A.A., Feijóo, R.A., Padra, C., Taroco, E.: Topological sensitivity analysis. Comput. Meth. Appl. Mech. Eng. 192(7-8), 803-829 (2003)

23. Laurain, A.: Singularly Perturbed Domains in Shape Optimisation. Phd thesis, Université Henri Poincaré, Nancy (2006).

24. Novotny, A.A., Feijóo, R.A., Taroco, E., Padra, C.: Topological sensitivity analysis for threedimensional linear elasticity problem. Comput. Meth. Appl. Mech. Eng. 196(41-44), 4354-4364 (2007)

25. Hlaváček, I., Novotny, A.A., Sokołowski, J., Żochowski, A.: On topological derivatives for elastic solids with uncertain input data. J. Optimiz. Theory Appl. 141(3), 569-595 (2009)

26. Khludnev, A.M., Novotny, A.A., Sokołowski, J., Żochowski, A.: Shape and topology sensitivity analysis for cracks in elastic bodies on boundaries of rigid inclusions. J. Mech. Phys. Solids 57(10), 17181732 (2009)

27. Ammari, H., Kang, H.: Polarization and Moment Tensors with Applications to Inverse Problems and Effective Medium Theory. Applied mathematical sciences. Springer-Verlag, New York (2007)

28. Nazarov, S.A., Sokołowski, J., Specovius-Neugebauer, M.: Polarization matrices in anisotropic heterogeneous elasticity. Asymptot. Anal. 68(4), 181-221 (2010) 